\title{
Near-Field Fluorescence Imaging of Genetic Material: Toward the Molecular Limit
}

\author{
N. F. van Hulst, M. F. Garcia-Parajo, M. H. P. Moers, J .-A. Veerman, and A. G. T. Ruiter \\ Applied Optics Group and MESA Research Institute, Department of Applied Physics, University of Twente, \\ P.O. Box 217, 7500 AE Enschede, The Netherlands
}

Received March 17, 1997, and in revised form April 21, 1997

\begin{abstract}
Chromosomes, DNA, and single fluorescent molecules are studied using an aperture-type near-field scanning optical microscope with tuning fork shear force feedback. Fluorescence in situ hybridization labels on repetitive and single copy probes on human metaphase chromosomes are imaged with a width of $80 \mathrm{~nm}$, allowing their localization with nanometer accuracy, in direct correlation with the simultaneously obtained topography. Single fluorophores, both in polymer and covalently attached to amino-silanized glass, are imaged using two-channel fluorescence polarization detection. The molecules are selectively excited according to their dipole orientation. The orientation of the dipole moment of all molecules in one image could be directly determined. R otational dynamics on a 10-ms to 100-s timescale is observed. Finally, shear force imaging of double-stranded DNA with a vertical sensitivity of $0.2 \mathrm{~nm}$ is presented. A DNA height of $1.4 \mathrm{~nm}$ is measured, which indicates the nondisturbing character of the shear force mechanism. 1997 Academic Press
\end{abstract}

\section{INTRODUCTION}

In the last decade, scanning probe microscopy has emerged as a useful tool in biological research. Scanning force microscopy (SFM) especially has demonstrated nanometer-scale resol ution on biological samples at native conditions. Processes of molecular assembly in real time and near-physiological environments can be readily followed within the spatial and temporal limitations imposed by a SFM experiment (Radmacher et al., 1992, 1994; Putman et al., 1994; Guthold et al., 1994). However, despite the outstanding vertical and lateral sensitivity of force sensing, optical detection has remained essential to biological investigation due to its convenience and noninvasiveness and the extensive variety of contrast mechanisms associated with light. In particular, the chemical specificity contained in spectro- scopic information is vital for the understanding of many biological processes. Unfortunately, the spatial resolution in conventional optical microscopy is limited to about half the optical wavelength, i.e., the diffraction limit. Imaging of optical contrast at nanometer scale requires the detection of the nearfield optical interaction, involving nonpropagating waves at a subwavelength scale. The feasibility of near-field optics was explored experimentally immediately following the start of scanning probe microscopy (SPM) in 1981, even before SFM (Pohl et al., 1984). Yet among the SPM techniques near-field optics has long been considered an academic peculiarity, especially compared to the enormous impact of SFM. Only in recent years with improving efficiency (Betzig et al., 1991) and versatility has near-field optics started showing its latent promises of "optical contrast" at nanometer dimensions and the steadily growing number of biological applications is indicative of its future importance (van Hulst and Moers, 1996).

In near-field scanning optical microscopy (NSOM) a probe consisting of a very small (subwavelength dimension) aperture is generally scanned in close proximity (within the near field, i.e., $<10 \mathrm{~nm}$ ) to the specimen under study. Using the probe as a nearfield excitation source, the interaction with the samplesurface induces changes in the far-field radiation, which is collected by conventional optics and directed to a highly sensitive detector to provide an optical image (Pohl et al., 1984; Betzig et al., 1991). An independent mechanism is used to control the distance separation between the probe and the sample within 1 to $10 \mathrm{~nm}$ and generate simultaneously a topographic image (Toledo-Crow et al., 1992; van Hulst et al., 1993). In this way, a singular feature pertaining to NSOM is produced: correlative optical and topographical imaging with a spatial resolution determined by the probe configuration. Another unique characteristic of near-field excitation is given by the finite size of the probe itself: decreasing the 
area of illumination obviously reduces the interaction volume and background scatter, which is of major importance in enhancing the sensitivity in spectroscopicapplications (fluorescence, Raman, etc).

The development of ultrasensitive detectors ( $\mathrm{Li}$ and Davis, 1993) combined with the high background rejection afforded by efficient near-field optical probes (Betzig et al., 1991) has allowed the detection and imaging of single molecular fluorescence over the last 3 years (Betzig and Chichester, 1993; Xie and Dunn, 1994; Ambrose et al., 1994; Ruiter et al., 1997b). Simultaneously, topographic imaging in NSOM is approaching the quality of regular SFM through refinements of probe-sample distance control regulation (Karrai and Grober, 1995). All these advances allow us to readily envisage the challenge of near-field optical studies of biological processes at the molecular level.

In this paper we present advances toward the molecular level in near-field fluorescence imaging of genetic material. Starting with human metaphase chromosomes, labeled by fluorescence in situ hybridization, we demonstrate simultaneous topographic and near-field fluorescence imaging, with a resolution of $80 \mathrm{~nm}$. Next we move down to the level of singlefluorophore detection, showing molecular localization with nanometer accuracy and direct determination of the molecular dipole orientation. Finally, shear force imaging of DNA in a NSOM with a vertical sensitivity of $0.2 \mathrm{~nm}$ is presented.

\section{EXPERIMENTAL SETUP}

The low density of objects per unit area is one of the practical problems encountered when investigating a biological sample with a SPM. For example, in the preparation of human metaphase chromosomes only a few metaphases of sufficient quality are found on a square millimeter. Consequently, it is important to integratea SPM in a conventional high-magnification optical microscope for fast location and preselection of a specific area of interest. Moreover, it is practical to design the SPM such that standard object glasses or coverslips can be accommodated. For fluorescence imaging of single molecules, efficient optical signal detection and background rejection, combined with long-term stability and high spatial resolution, is required. At the same time, mechanical, electronic, and acoustic noises have to be minimized ( $<0.1 \mathrm{~nm}$ vertically) if topographical molecular resolution is pursued. To satisfy all the above requirements, we built our NSOM into a Zeiss Axiovert 135 TV inverted optical microscope, which was chosen for its high mechanical stability and great flexibility. Additionally, during experiments the mi croscope head is encl osed to minimize acoustic noise and the whole system is suspended to reduce unwanted mechanical vibrations.

The experimental setup is schematically shown in Fig. 1. A near-field optical light source consisting of a tapered, aluminium-coated single mode optical fiber (Newport F-SV) with an aperture of less than $100 \mathrm{~nm}$ is used to illuminate the sample. The power of an incident $\mathrm{Ar}^{+} / \mathrm{Kr}^{+}$laser is set such that the excitation power at the end of the coated fiber is about $3 \mathrm{nW}$, as measured in the far field. The sample is scanned underneath the fiber and the fluorescence emission from the sample plane is collected with a high NA objective (0.75 NA dry or 1.3 NA immersion oil) and directed to one or two detectors depending on the particular experiment. The fluorescence is filtered

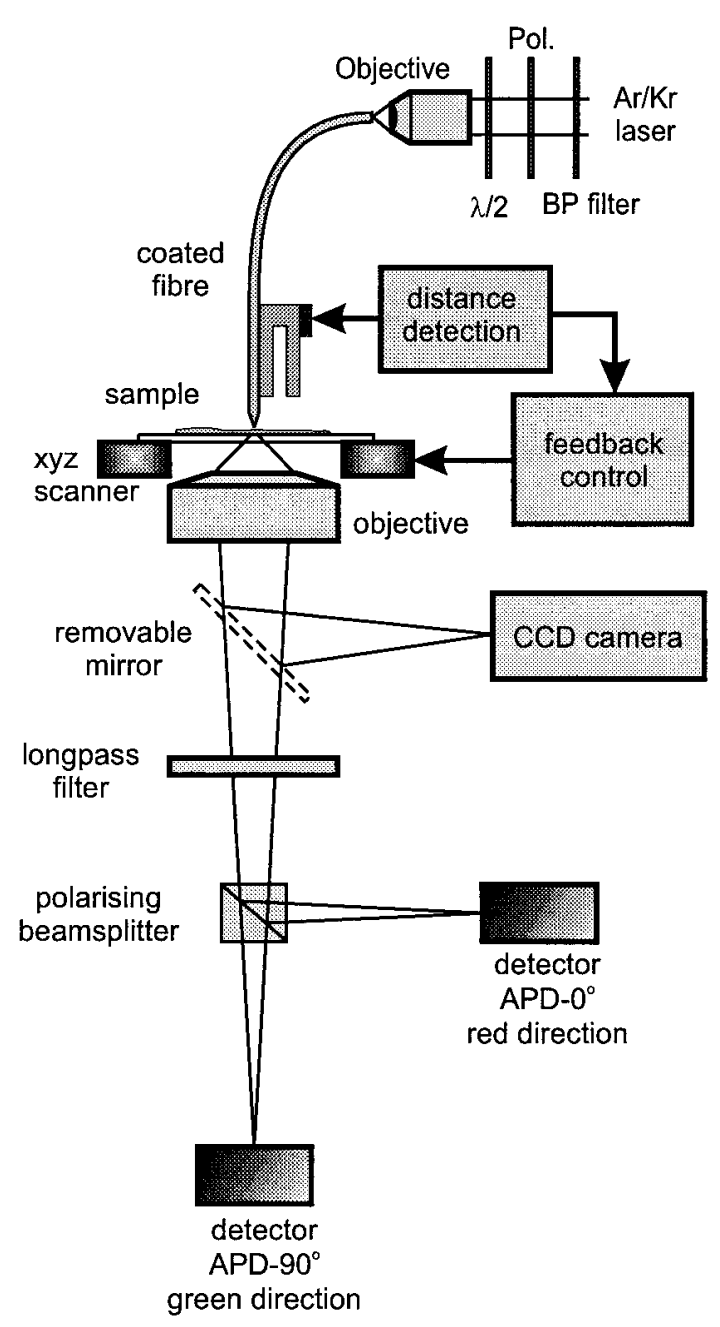

Fıg. 1. Schematic setup of the aperture-type near-field scanning optical microscope (NSOM) with a metal-coated tapered fiber. The scanning sample stage is mounted on top of an inverted optical microscope. Shear force feedback has been implemented to control the tip-sample distance separation. Fluorescence is collected in transmission using a high NA objective, split into two perpendicular polarization directions by a polarizing beam splitter and detected with two avalanche photodiodes (APD). 
from the transmitted light using a long-pass filter $(\lambda>550 \mathrm{~nm})$. Photon counting avalanche photodiodes (APD, SPCM-100 from EG\&G Electro Optics) are used as detectors. In the near-field operation the fiber aperture is confocally aligned onto the detectors. Shear force phase feedback based on a quartz tuning fork system (Ruiter et al., 1997a) is used to maintain a constant tip-sample separation and to generate high-resolution topographical images. A personal computer controls the raster scanning of the sample using two D/A converters and collects the counts per pixel for each detector.

For single molecular fluorescence experiments, particularly in the study of rotational diffusion and reorientation of individual molecules within the plane of the sample, we make use of the two APD detectors. In this case, the fluorescence signal is separated into its two perpendicular polarization directions using a broadband polarizing beam splitter cube (Newport, 400-700 nm). By monitoring the relative contributions of both polarization components, the in-plane molecular dipole orientation can be determined. Also, a polarizer and a rotatable $\lambda / 2$ plate have been placed before the coupling of the light into the fiber to allow rotation of the excitation polarization. The resulting polarization extinction ratio of the near-field probe is generally better than 10 , as determined in the far field.

\section{FLUORESCENCE IN SITU HYBRIDIZATION OF HUMAN CHROMOSOMES}

Chromosome structure has been investigated with all microscopical techniques available. In addition to the pure morphological examination of chromosomes, specific information on the DNA sequence can be correlated to the chromosome structure by the use of labeling techniques, specifically in situ hybridization as developed almost 30 years ago ( $\mathrm{J}$ ohn et al., 1969; Gall and Pardue, 1969). After the early practice of radioactive labels (Buongiorno-Nordelli and Amaldi, 1970) the more practical detection of in situ hybridized DNA, on the basis of fluorescence labels or enzyme-generated dyes, has promoted fluorescence in situ hybridization (FISH) to one of the major cytogenetic detection methodologies for human genetics (Rudkin and Stollar, 1977; Lichter et al., 1991). FISH enables direct visualization of topological or positional information about gene sequences in a fluorescence microscope, allowing rapid localization of genomic DNA fragments in morphologically preserved inter- and metaphase chromosomes. A resolution better than $1 \mathrm{Mb}$ can be obtained using (pro)metaphase chromosomes (Wiegant et al ., 1992), while $1 \mathrm{~kb}$ is feasible on stretched DNA. Yet the localization of the fluorescence labels is fundamentally limited to $\sim 300 \mathrm{~nm}$ by diffraction in conven- tional fluorescence microscopy, while localization of the numerous closely linked genes requires mapping at higher resolution. Using electron microscopy, immunogold DNA probes can be imaged with nanometer resolution. Fetni et al. (1991) demonstrated localization of single copy DNA sequences in subbands of prophase chromosomes using immunogold labeling. Putman et al. (1993) pioneered the potential of SFM in the detection of morphological in situ hybridization labels, assuming that cytochemical staining techniques yield specific topological features that can be discriminated from the surrounding chromosomal corrugation. I ndeed, after enhancement by an enzymatic reaction, morphological labels of 75-100 nm in diameter could be discriminated, but unique single copy DNA targets could not yet be observed. Despite the higher resolution of electron and force microscopy they lack the multiplicity of fluorescence detection. In fact, the widespread use of FISH is mainly due to its unparalleled specificity afforded by the potential of multicolor labeling (up to 6) in one preparation (Wiegant et al., 1993). In this paper we show that near-field fluorescence microscopy combines the best of both worlds: optical resolution beyond the diffraction limit, combined with nanometer topographic resolution in simultaneous force microscopy, and multiplicity through multicolor fluorescence detection with sensitivity down to the single molecular level. Near-field fluorescence images of single copy and repetitive FISH labels on human metaphase chromosomes are presented, together with the chromosomal morphology as obtained by shear force imaging.

Human metaphase chromosome spreads were prepared on microscope coverslips from a phytohemagglutinin-stimulated primary peripheral blood lymphocytecultureaccording to routine procedures. Two types of repetitive DNA probes, specific for chromosome No. 1, were used: the satellite III probe pUC1.77, which recognizes the near-centromeric region (1q12), and the probe p1-79, which is specific for the telomere region of the short arm, with insert sizes of 1.77 and $0.90 \mathrm{~kb}$, respectively. Also, the plasmid probe $\alpha$-spectrin was used, a unique DNA probe that hybridizes specifically at 1q21.3, with an insert length of $13 \mathrm{~kb}$. The probes were labeled with either biotin or digoxigenin by nick translation. The biotinated pUC-1.77 is indirectly detected by fluorescein isothiocyanate (FITC, a green fluorescent dye), while digoxigenin-labeled p1-79 and biotinated $\alpha$-spectrin are detected by cyanine (CY-3, an orange fluorescent dye). The chromosome spreads are dried in air for imaging purposes. The fluorescence quantum efficiency of $\mathrm{CY}-3$ is high (>0.8) in both buffer and air; however, FITC is significantly quenched in air.

Figures $2 \mathrm{a}$ and $2 \mathrm{~b}$ and $\mathrm{c}$ demonstrate two-color 
fluorescence in situ hybridization to a human metaphasechromosome No. 1: p1-79 hybridized to 1p36 in the telomeric region, with orange CY-3 staining, and pUC1.77 hybridized to $1 q 12$ in the centromeric area, with green FITC staining (Moers et al., 1996). The image is scanned with a $35-\mathrm{nm}$ pixel size, a 40 ms/pixel scan speed, blue excitation (473 $\mathrm{nm} \mathrm{Ar}^{+}$ line) for FITC, and green excitation (521 nm Kr+ line) for CY-3. In the shear force image (Fig. 2a) the piezo feedback signal is displayed with slight horizontal high spatial frequency filtering. The well-known metaphase chromosomal structure with well-separated chromatides is clearly resolved with details as small as $40 \mathrm{~nm}$ and a height up to $\sim 150 \mathrm{~nm}$. The corresponding near-field optical image (Fig. 2b) displays the orange fluorescence at $\lambda>550 \mathrm{~nm}$, using BG39 and KV550 Schott filters. The p1-79 probes are visualized as distinct substructures in the telomere region, with at least five probes in each chromatide (maximum 700 counts/pixel). Apart from the bright signals at the telomere, several isolated weak spots ( $\sim 50$ counts/pixel) are detected on the chromatides in the centromeric region. Figure $2 c$ displays the green fluorescence at $\lambda>500 \mathrm{~nm}$, corresponding mainly to the FITC-stained pUC-1.77 probe in the centromeric area. Indeed, a large centromeric area fluoresces, but additional signals show up at the same positions as the $\mathrm{CY}-3$ signals in $\mathrm{Fig}$. $2 \mathrm{~b}$. Inspection by eye with the inverted microscope reveals that these spots are orange. Obviously upon blue excitation at $\lambda=473 \mathrm{~nm}$ the efficient fluorophore $\mathrm{CY}-3$ is also excited, giving some cross-talk between the channels. Autofluorescence can be recognized as a weak general background signal ( $\sim 5$ counts/pixel) over the total chromosome area, especially upon blue excitation.

From a cytochemical point of view the location of single copy genes, which recognize unique DNA targets, is especially important. Near-field fluorescence imaging of a unique DNA probe is presented in Figs. 2d and 2e: $\alpha$-spectrin hybridized specifically to $1 q 21.3$ at the long arm and p1-79 cohybridized to 1 p36 in the telomeric region, both with orange CY-3. Figure $2 \mathrm{~d}$ shows the topography and Fig. $2 \mathrm{e}$ the corresponding near-fiel d fluorescence image showing the unique probe $\alpha$-spectrin as two single fluorescent spots at the internal side of both chromatide bands (top) and again the multiple CY-3 FISH labels at the tel omere probe p1-79 (bottom). Clearly, the combined forceand fluorescenceimages al low accurate determination of the probe location on the chromosome morphology. Generally the $\alpha$-spectrin is observed at the external side of the chromatides, both in our near-field studies and in conventional fluorescence microscopy, with an occasional exception, as in Fig. 2e. In fact, upon detailed inspection of the force image a twist is observed in the centromeric area of the chromosome, which possibly explains the internal location of the FISH signals in this specific case.

Figure 3 shows a 1- $\mu$ m line trace through two very weak CY-3 labels as observed in the centromeric area in Fig. 2b. Both fluorescence spots have a width (FWHM) of $80 \mathrm{~nm}$, while clearly separated at a distance of only $110 \mathrm{~nm}$. The line trace clearly indicates the superior optical resolution provided by NSOM, including the ability to localize the fluorescence maximum with an accuracy of a few nanometers. The signal level of the fluorophores in Fig. 3 corresponds to $\sim 1000$ counts/sec, which is estimated to originate from fewer than $10 \mathrm{CY}-3$ molecules. Obviously, the detection of single molecular labels is the next challenge.

\section{SINGLE MOLECULAR FLUORESCENCE DETECTION}

The observation of individual molecules has always represented the ultimate goal in fluorescence detection sensitivity. To date, imaging of single molecules under ambient conditions with high spatial and temporal resolution is readily achievable (Xie and Dunn, 1994). Further advances in the field show that single molecule experiments do provide a sensitive tool for studying the local environment of a single molecule under ambient conditions (Dunn et al., 1994). The exact determination of the molecular dipole orientation is likewise interesting because many dynami c processes, such as protein and molecular conformational changes, can be studied by tracking the particular orientation of the molecules. Changes on the dipole orientation are associated with particular biomolecular events (Vamosi et al., 1996). For instance, the exactness of fluorescence energy transfer experiments between a single donoracceptor pair depends critically on the determination of the molecular dipole orientation (Ha et al., 1996a). In addition, if selective excitation of molecules according to their particular orientation is achieved, it can then be exploited as a tool for triggering or inhibiting specific biological reactions.

At present, in the near field, polarization contrast techniques have shown the ability to distinguish between regions of different molecular dipol e orientation (Moers et al., 1994; Higgins et al., 1996). In the far field, Ha et al. (1996b) have recently monitored single molecular rotational dynamics using excitation polarization modulation.

The obvious next step in the study of conformational and structural changes in DNA and proteins is to follow dynamic processes such as diffusive lateral and rotational motions on single molecular bases. In order to observe single molecular rotational and translational diffusion we have extended our nearfield scanning optical microscope with two polariza- 

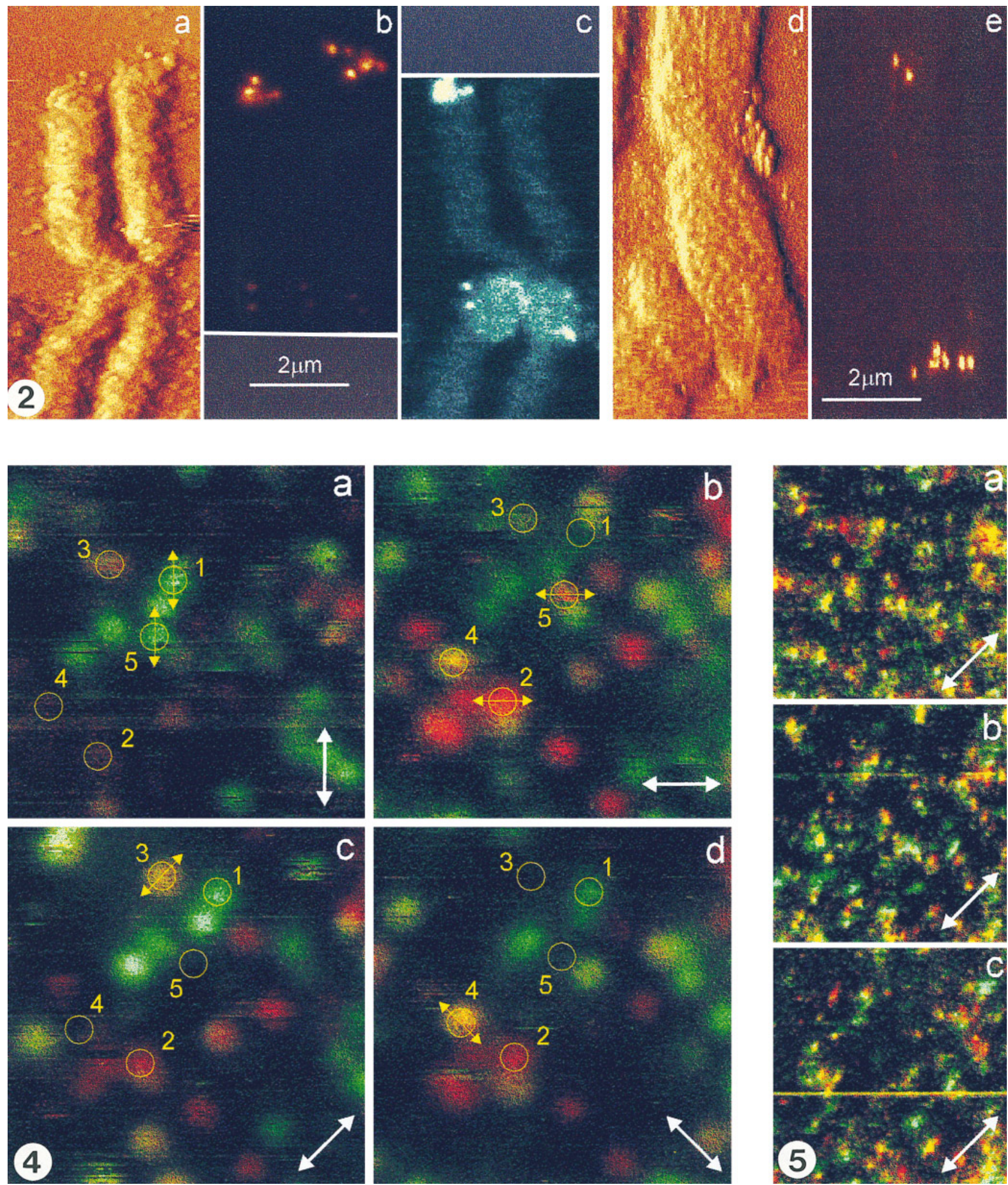

Fıg. 2. (a,b,c) Two-color fluorescence in situ hybridization to human metaphase chromosome No. 1 (image size $4 \times 7 \mu m^{2}$ ): (a) Shear force image (slightly high pass filtered in the horizontal direction), displaying the chromosome topography with a height up to $\sim 150 \mathrm{~nm}$; (b) corresponding near-field fluorescence image at green excitation ( $\lambda=521 \mathrm{~nm}$ ) showing orange $(\lambda>550 \mathrm{~nm}) \mathrm{CY}-3 \mathrm{FISH}$ labels at the telomere probe p1-79 (top) and additional probes in the centromeric region; (c) sequential near-field fluorescence image at blue excitation $(\lambda=473 \mathrm{~nm}$ ) showing green FITC FISH labels at the centromeric probe pUC-1.77 (Moers et al., 1996). (d,e) Near-field fluorescence of a unique DNA probe (image size $4 \times 7 \mu \mathrm{m}^{2}$ ): (d) topography (note the twist in the centromeric area of the chromosome); (e) corresponding near-field fluorescence image showing orange CY-3 FISH labels at the telomere probe p1-79 (bottom) and the unique probe $\alpha$-spectrin as single fluorescent spots at the inside of the chromatide bands (top). 


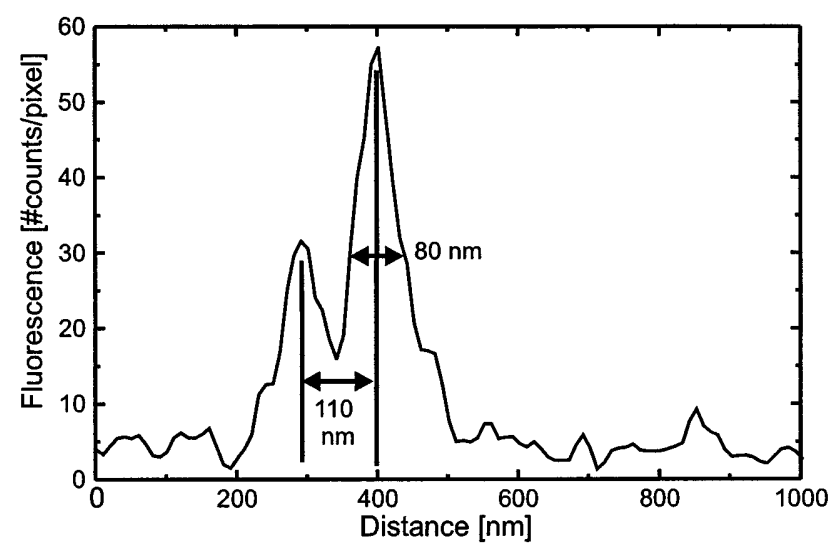

FIG. 3. Near-field fluorescence line trace $(1 \mu \mathrm{m})$ through in situ hybridized human chromosome No. 1. Two CY 3 labels separated by $110 \mathrm{~nm}$ are shown. The FWHM is $80 \mathrm{~nm}$ in both cases. Fluorescence photocounts are integrated over $40 \mathrm{msec}$.

tion detection channels (Ruiter et al., 1997b; GarciaParajo et al., 1997). Some recent results on polarization-sensitivesingle molecular detection of fluorophores both in polymer and bound to aminosilanized glass are presented.

Samples of carbocyanine (Dil- $\mathrm{C}_{18}$ ) dye molecules embedded in a thin polymethylmethacrylate (PMMA) layer were prepared in the following way: Dil (Molecular Probes, D-282) molecules were diluted in methanol (Merck, 99.8\%) to a final concentration of $5 \times 10^{-8} \mathrm{M}$. The dye solution was then added into a $0.5 \%$ weight PMMA in chloroform (Merck, 99.4\%). A 10- to 20- $\mu$ l drop was deposited onto a freshly cleaned $170-\mu \mathrm{m}$-thick glass coverslip and spin coated at $4000 \mathrm{rpm}$. The resulting layer thickness was between 5 and $10 \mathrm{~nm}$, with a surface coverage of typically a few dye molecules per square micrometer.

Figure 4 shows four images of individual molecules over the same area. The image size is $3 \times 3$ $\mu \mathrm{m}^{2}$, with a pixel integration time of $10 \mathrm{msec}$. Each image corresponds to a specific excitation polarization $\left(a, 90^{\circ} ; b, 0^{\circ} ; c, 45^{\circ}\right.$; and $\left.d,-45^{\circ}\right)$ with respect to the line scan direction. Although Fig. $4 \mathrm{a}$ is slightly shifted with respect to the others, corresponding molecules can unambiguously be identified in the consecutive scans. Each image consists of two data sets, one for each fluorescence polarization direction. The data have been color coded, red for the $0^{\circ}$ detector and green for the $90^{\circ}$ detector, and added up to create one image. As a result, the color of each image reflects the relative contribution of each polarization component (i.e., equal amounts of red and green give yellow), while the brightness reflects the total fluorescence intensity. In what follows, the two polarization directions will bereferred to as the "red" and "green" directions. Close inspection of Fig. 4 reveals different distributions of molecules in each image. Some molecules which are present in one image are absent in the other. Image (a) displays mainly green colored molecules, as the excitation was parallel to the green detection channel. Similarly, image (b), with the excitation rotated $90^{\circ}$ to the red detection channel, shows mainly red molecules. In images (c) and (d) some bright yellow molecules show up, excited at $45^{\circ}$ and $-45^{\circ}$, respectively. Clearly, with this set of data the molecular dipole moment can be determined. It is concluded that absorption and emission moments are about parallel. Thein-plane dipole orientation of somecharacteristic molecules is marked in Fig. 4: molecules numbered 1 to 4 are oriented close to $90^{\circ}, 0^{\circ}, 45^{\circ}$, and $-45^{\circ}$, respectively. The color of the majority of the molecules remained constant during imaging, which excludes fast rotation. Yet some dynamics are also observed. For example, molecule 5 is oriented close to $90^{\circ}$ in Fig. $4 \mathrm{a}, 0^{\circ}$ in Fig. $4 \mathrm{~b}$, and has disappeared in Figs. $4 \mathrm{c}$ and $4 \mathrm{~d}$, probably due to photodissociation. Other molecules cease emitting over a few pixels or even several scan lines, to reappear with their initial intensity and polarization. These observations are attributed to a temporary molecular transition to a dark nonemitting state, while retaining the dipole orientation.

Confident that we were able to investigate single molecular fluorescence with our NSOM, we directed our efforts toward the detection of amino-reactive fluorophores known to bind covalently to specific sites of amino-modified oligonucleotides. As a first step on the study of DNA-dye interactions, we attempted the detection of individual tetramethylrhodamine (TRITC) molecules attached to an aminosilanized substrate. The attachment of the TRITC to this particular substrate mimics the binding of TRITC to the oligonucleotide via an amino group at

Fıg. 4. Near-field polarized fluorescence images of individual carbocyanine $\left(\mathrm{Dil}-\mathrm{C}_{18}\right)$ molecules over the same area $\left(3 \times 3 \mu \mathrm{m}^{2}\right)$, each with different excitation polarization relative to the line scan direction: (a) $90^{\circ}$; (b) $0^{\circ}$; (c) $45^{\circ}$; and (d) $-45^{\circ}$, as indicated by arrows. The images are color-coded in red and green, corresponding to $0^{\circ}$ and $90^{\circ}$ polarized fluorescence, respectively. The dipole orientation of molecules numbered 1 to 4 is close to $90^{\circ}, 0^{\circ}, 45^{\circ}$, and $-45^{\circ}$, respectively. Molecule 5 is oriented close to $90^{\circ}$ in (a) and rotates to $0^{\circ}$ in (b).

FIG. 5. Sequential near-field fluorescence images $\left(3 \times 3 \mu \mathrm{m}^{2}\right)$ at $15 \mathrm{~min}$ time interval $(\mathrm{a}-\mathrm{c})$ of tetramethylrhodamine (TRITC) mol ecules covalently attached to an amino-silanized glass. Excitation polarization at $\sim 45^{\circ}$ relative to the detection channels, as indicated by arrows. The images are color-coded in red and green, corresponding to $0^{\circ}$ and $90^{\circ}$ polarized fluorescence, respectively. Preferentially, molecules around $45^{\circ}$ in-plane dipole orientation are excited, showing up as yellow. 
the end of the coupled carbon linker to the $5^{\prime}$ end of the DN A strand. Samples were prepared by depositing a $5-\mu$ l drop of the dye solution on amino-silanized glass. A cleaned coverslip was carefully placed on top of the modified substrate, with the solution between the two surfaces. The drop spread immediately as the coverslip and the amino-silanized glass sealed together. The sandwiched solution was then incubated in the dark at $4^{\circ} \mathrm{C}$ for $2 \mathrm{hr}$. Finally, the coverslip was removed, and the sample was thoroughly rinsed with PBS and air-dried before imaging. As dye solution we used a final concentration of 15 nM 5-isomer of TRITC (Molecular Probes, T-1480) diluted in high-purity DMSO. The amino-silanized glass was prepared using (aminopropyl)triethoxysilane (Aldrich, 98\%) with a method similar to one developed by Karrasch et al. (1993).

Figure 5 shows three images (a-c) of bound TRITC molecules, again in polarization red/green color coding, taken at 15-min time intervals, with excitation polarization adjusted at $\sim 45^{\circ}$ relative to the detection channels. Theimage size is $3 \times 3 \mu^{2}(200 \times 200$ pixels), with a pixel integration time of $10 \mathrm{msec}$. Individual TRITC molecules can be distinguished, despite the lower signal-to-noise ratio compared to the case of Dil or R-6G molecules. The poorer discrimination of the molecules can be attributed to their lower quantum efficiency $(0.28$, compared to 0.90 for Dil), especially when conjugated to amino groups (Tsien and Waggoner, 1990). Molecules around $45^{\circ}$ in-plane dipole orientation are preferentially excited, showing up as yellow in the Fig. 5. Detailed inspection reveals that the stability of the molecular orientation varies from case to case, regardless of the binding to the surface: some rotate within one scan, others are stable over an hour. Photobleaching of bound TRITC molecules was observed to occur more rapidly than in the case of Dil in polymer, despite the lower emission rate. We noticed that during the first scan approximately half of the molecules bleached (compare Figs. 5a and 5b), while the surviving molecules experienced a longer lifetime, fluorescing typically another four consecutive images before photodissociation. This observation of the bimodal photobleaching behavior of TRITC has been also reported by Schmidt et al. (1995). Currently, we are investigating alternative dyes that bind selectively to the DNA strands, with high photostability and quantum efficiency.

These results illustrate the capability of our setup for selective excitation and detection of single molecules according to their absorption and emission dipoleorientation. Thein-plane orientation of stationary molecules can be determined with an accuracy of a few degrees. Rotational dynamics on a 10-msec to $100-\mathrm{sec}$ timescale is readily visualized. Moreover, the location of maximum fluorescence of the molecule can be determined with an accuracy of $\sim 1 \mathrm{~nm}$. The imaging of single covalently bound aminoreactive fluorophores is a first step toward detection of oligonucleotide probes.

\section{SHEAR FORCE DNA IMAGING}

The single molecular fluorescence capability of NSOM has to be complemented with molecular resolution topographical imaging for unambiguous localization of fluorophores. As mentioned, shear force detection has proven to be a very sensitive method for distance regulation (feedback) and topographical imaging in NSOM, despite unclearness about the actual shear force interaction mechanism. We have improved our shear force sensitivity using a quartz piezoelectric tuning fork as a resonant sensor mounted to the fiber probe (Ruiter et al., 1997a). The resonance frequency of the combined tuning forkNSOM tip system is typically 33 to $34 \mathrm{kHz}$. The system is driven externally with an oscillation amplitude of 14 pm peak-to-peak, corresponding to about $2.5 \mathrm{~nm}$ lateral displacement of the tip, as a result of the resonance amplification with a Q-value of 175 . The bandwidth of the feedback regulation has been enhanced to a few hundred hertz by detecting the phase of the tuning fork response. The phase set point is chosen to be close to the out-of-contact value. A vertical sensitivity of $0.2 \mathrm{~nm}$ is obtained. The scan speed is about $1 \mu \mathrm{m} / \mathrm{sec}$, comparable to the value for single molecule NSOM imaging. The performance of this shear force microscope in imaging of DNA is demonstrated.

The DNA sample was prepared in the following way: $10 \mathrm{ng} / \mu \mathrm{l}$ of 600 - to $800-\mathrm{bp}$-long double-stranded DNA fragments was diluted in $10 \mathrm{mM}$ Hepes, $\mathrm{pH} 7.4$, and $1 \mathrm{mM} \mathrm{ZnCl}$, giving a final concentration of 1-5 $\mathrm{ng} / \mu \mathrm{l}$ for deposition. A 10- to $20-\mu \mathrm{l}$ drop was deposited onto freshly cleaved mica. After the sample settled for a couple of minutes, it was washed with water $(0.5 \mathrm{ml}$ of doubly distilled water from a squirt bottle) and then briefly dried in a stream of compressed air before imaging.

Figure 6 shows shear force images of doublestranded DNA fragments as deposited on mica, using a freshly pulled optical fiber tip. The images show clearly resolved strands, 200-300 nm in length, with a lateral width of $24 \pm 2 \mathrm{~nm}$ at half-height and a height of $1.4 \pm 0.2 \mathrm{~nm}$, as inferred from cross sections at the DNA filaments. While the lateral resolution is slightly worse than that of SFM measurements, the DNA height is larger than the value of 0.5-0.7 nm reported with SF M techniques (Bustamante et al., 1992; Bezanilla et al., 1995; Schaper et al., 1994) and much closer to the $2 \mathrm{~nm}$ expected for $B-D N A$ in solution. The reduced DNA height in SFM 
imaging has been commonly attributed to indentation of the DNA (Wyman et al., 1995), adhesion effects by the contacting tip (van Noort et al., 1997), and the dehydration of the DNA in the air-dried samples (Pietrasanta et al., 1994). In our case, we believe that only dehydration may have played a role on the reduced measured height since the tip makes no physical contact with the sample. Furthermore, we did not notice any degradation nor incisions on the DNA strands even after repetitive scanning of the same area.

Figure 7 shows another example of the sensitivity of our technique. In this case, we have used the standard aluminium-coated NSOM tip for imaging. Despite a decrease on the lateral resolution, probably caused by the grainy structure of the metal coating, the vertical sensitivity remains around 0.2 $\mathrm{nm}$ (from the surface plot the flatness of the mica
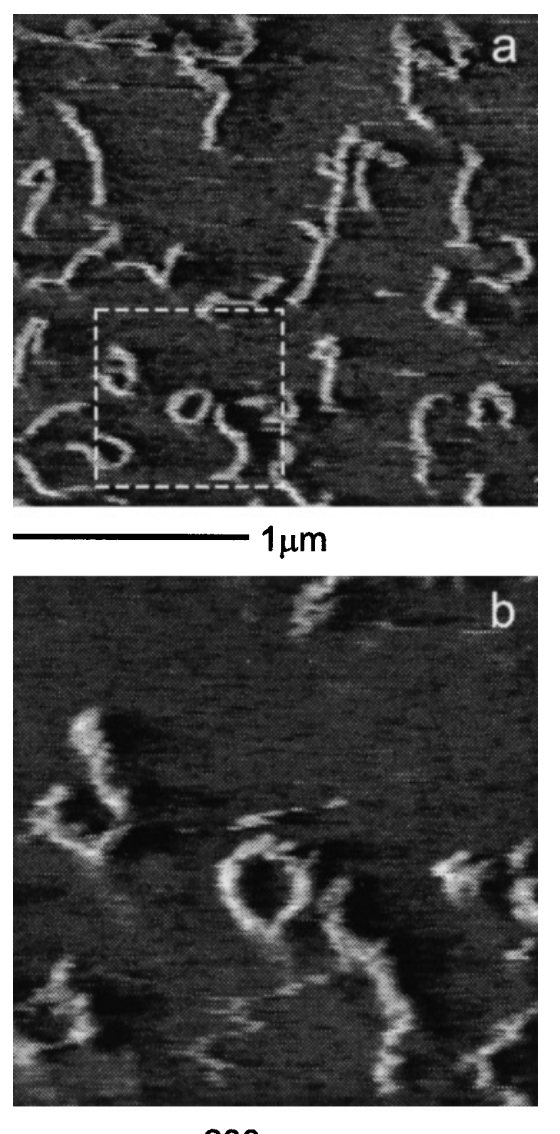

$200 \mathrm{~nm}$

Fıg. 6. (a) A $2.2 \times 2.2 \mu \mathrm{m}^{2}$ shear force image of doublestranded DNA fragments as deposited on mica, using an uncoated fiber tip. DNA filaments, 600 to $800 \mathrm{bp}$ long, with a lateral width of $24 \pm 2 \mathrm{~nm}$ and a height of $1.4 \pm 0.2 \mathrm{~nm}$ are observed. (b) A $600 \times$ $600 \mathrm{~nm}^{2}$ scan of the area marked in (a); note the mobility of some DNA fragments caused by the loose binding to the mica, achieved by adjusting the ionic concentration of the buffer during the solution preparation.

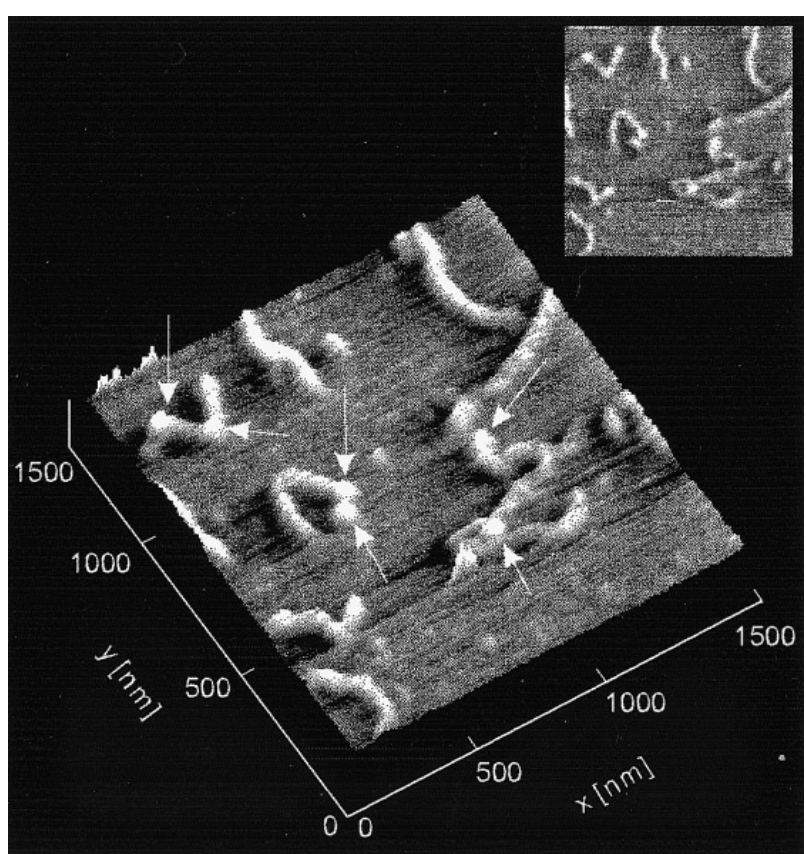

FIg. 7. Surface plot $\left(1.5 \times 1.5 \mu \mathrm{m}^{2}\right)$ of a shear force image, using an aluminium-coated NSOM tip, of double-stranded DNA fragments combined with globular proteins (40-kDa monomer), as indicated by the arrows. The protein and DNA were allowed to form complexes in a standard in vitro binding reaction before deposition for imaging. The vertical sensitivity of $\sim 0.2 \mathrm{~nm}$ is clearly seen in the flatness of the mica surface.

surface is clearly seen). In fact, we are able to distinguish globular proteins (purified mouse XPA) as small as a 40-kDa monomer in different parts of the DNA fragments, as indicated by arrows in Fig. 7. The identification and specific localization of XPA along the DNA strands using shear force imaging allows recognition of DNA damaged sites, a first step on DN A repair studies (Wyman, 1997).

These results demonstrate that shear force imaging is an interesting alternative technique for highresolution imaging of soft material and in particular for the study of DNA-protein interactions that re quire "loose" immobilization of the DNA onto the flat surface. The lateral resolution will depend mainly on the residual lateral oscillation amplitude and the tip radius of the fiber probe, which can in principle be made down to a few nanometers (Garcia-Parajo et al., 1995).

\section{CONCLUSIONS}

We have shown that combined near-field fluorescence and shear force microscopy can be applied to imaging and studying genetic material. The technique brings together several advantages: (i) resolution beyond the diffraction limit and a reduced bleaching rate of fluorophores, compared to confocal microscopy, while retaining the advantages of multi- 
color detection and single molecule sensitivity; (ii) simultaneous topographical imaging with nanometric resolution using shear force feedback. Thus, due to the exquisite sensitivity of the instrument, mapping and colocalization studies of genetic material can be performed at an individual molecular level.

The detection of two-color fluorescence in situ hybridization signals on human chromosomes has been accomplished with an optical resolution of 80 $\mathrm{nm}$ and an accuracy of a few nanometers on the localization of the fluorescence maximum. The combination with force microscopy reveals valuable information on the associated morphological structure of the chromosome.

We have demonstrated single molecule fluorescence sensitivity using near-field optical microscopy. The molecules have been selectively excited according to their dipole orientation. The in-plane orientation of the molecular dipole could readily be determined for all molecules in a single image, using a two-channel polarization detection scheme. Single molecule rotational dynamics on a $10-$ msec to $100-$ sec timescale have been observed. The first applications to single fluorophore detection of an aminoreactive probe have been presented.

Shear force phase feedback, based on tuning fork detection, was used to obtain topographic imaging of protein-DNA complexes, with $0.2 \mathrm{~nm}$ vertical sensitivity. A double-stranded DNA height of $1.4 \pm 0.2 \mathrm{~nm}$ was measured, larger than the value reported with conventional SFM imaging. Because the shear force mechanism does not require physical contact with the specimen, degradation of the sample does not occur, making the technique an interesting alternative for the investigation of molecular events without invasion or disruption of the process itself.

In the near future we envisage combining topographic imaging of DNA with single molecular cytochemical sensitivity, i.e., approaching detection of a singleoligonucleotide probe. Weanticipate that many relevant biological and biophysical studies at the molecular level will be assessed with NSOM, such as sequencing and resequencing of DNA, recognition of point mutations, and study of the mutational repair process.

The authors thank Wouter Kalle, J oop Wiegant, Ton Raap, and Hans Tanke of Rijks Universiteit Leiden (NL) for the preparation of in situ hybridized chromosomes; Claire Wyman of Erasmus Universiteit Rotterdam (NL) and J ohn van Noort for DNA preparation and fruitful discussions; and Kees van der Werf, Frans Segerink, Wouter Rensen, I ne Segers, Margot Snel, Oscar Willemsen, and Bart de Grooth for their assistance and suggestions. Maria Garcia-Parajo is financed by the European HCM network on "Near-Field Optics for Nanoscale Science and Technology." J oost-Anne Veerman, Ton Ruiter, and Marco M oers are supported by the Dutch Foundation for Fundamental Research (FOM).

\section{REFERENCES}

Ambrose, W. P., Goodwin, P. M., Martin, J . C., and Keller, R. A. (1994) Alterations of single molecule fluorescence lifetimes in near-fiel d optical microscopy, Science 265, 364-367.

Betzig, E., Trautman, J. K., Harris, T. D., Weiner, J. S., and Kostelak, R. L. (1991) Breaking the diffraction barrier: Optical microscopy on a nanometric scale, Science 251, 1468-1470.

Betzig, E., and Chichester, R. J . (1993) Single molecule observed by near field scanning optical microscopy, Science 262, 14221425.

Bezanilla, M., Manne, S., Laney, D. E., Lyubchenko, Y. L., and Hansma, H. G. (1995) Adsorption of DNA to mica, silylated mica, and minerals: Characterization by atomic force microscopy, Langmuir 11, 665-659.

Buongiorno-Nordelli, N., and Amaldi, F. (1970) Autoradiographic detection of molecular hybrids between rRNA and DNA in tissue sections, Nature225, 946-947.

Bustamante, C., Vesenka, J ., Tang, C. L., Rees, W., Guthold, M., and Keller, R. (1992) Circular DNA-molecules imaged in air by scanning force microscopy, Biochemistry 31, 22-26.

Dunn, R. C., Holtom, G. R., Mets, L., and Xie, X. S. (1994) Near-field fluorescence imaging and fluorescence lifetime measurement off light harvesting complexes in intact photosynthetic membranes, J. Phys. Chem. 98, 3094-3098.

Fetni, R., Drouin, R., Lemieux, N., Messier, P.-E., and Richer, C.-L. (1991) Simultaneous visualisation of chromosome bands and hybridisation signal using colloidal-gold labelling in electron microscopy. Proc. Natl. Acad. Sci. USA 88, 10916-10920.

Gall, J . G., and Pardue, M. L. (1969) Formation and detection of RNA-DNA hybrid molecules in cytological preparations, Proc. Natl. Acad. Sci. USA 63, 378-383.

Garcia-Parajo, M. F., Tate, T., and Chen, Y. (1995) Gold-coated parabolic tapers for scanning near field optical microscopy: Fabrication and optimisation, Ultramicroscopy 61, 155-163.

Garcia-Parajo, M. F., Veerman, J .-A., Ruiter, A. G. T., and van Hulst, N. F. (1997) Single molecule dynamics observed with near-field optical microscopy, Ultramicroscopy Proceedings issue NF O4, N ovember 1997.

Guthold, M., Bezanilla, M., Erie, D. A., J enkins, B., Hansma, H. G., and Bustamante, C. (1994) Following the assembly of RNA polymerase-DNA complexes in aqueous solutions with the scanning force microscope, Proc. Natl. Acad. Sci. USA 91, 12927-12931. [and references therein]

Ha, T., Enderle, Th., Ogletree, D. F., Chemla, D. S., Selvin, P. R., and Weiss, S. (1996a) Probing the interaction between two single molecules: Fluorescence resonance energy transfer between a single donor and a single acceptor, Proc. Natl. Acad. Sci. USA 93, 6264-6268.

Ha, T., Enderle, Th., Chemla, D. S., Selvin, P. R., and Weiss, S. (1996b) Single molecule dynamics studied by polarization modulation, Phys. Rev. Lett. 77, 3979-3982.

Higgins, D. A., Vanden Bout, D. A., Kerimo, J ., and Barbara, P. (1996) Polarization-modulation near-field scanning optical microscopy of mesostructured materials, J. Phys. Chem. 100, 13794-13803.

van Hulst, N. F., Moers, M. H. P., and Bölger, B. (1993) Near-field optical microscopy in transmission and reflection modes in combination with force microscopy, J . Microsc. 171, 95-105.

van Hulst, N. F., and Moers, M. H. P. (1996) Biological applications of near-field optical microscopy, IEEE Eng. Med. Biol. 51-58.

J ohn, H., Birnstiel, M., and J ones, K. (1969) RNA-DNA hybrids at the cytological level, Nature 223, 582-587.

Karrai, K., and Grober, R. D. (1995) Piezo-electric tip-sample 
distance control for near field optical microscopes, Appl. Phys. Lett. 66, 1842-1844.

Karrasch, S., Dolder, M., Schabert, F., Ramsen, J ., and Engel, A. (1993) Covalent binding of biological samples to solid supports for scanning probe microscopy in buffer solution, Biophys. J . 65, 2437-2446.

Li, L-Q., and Davis, M. (1993) Single photon avalanche diode for single molecule detection, Rev. Sci. Instrum. 64, 1524- 1529.

Lichter, P., Boyle, A. L., Cremer, Th., and Ward, D. C. (1991) Analysis of genes and chromosomes by non-isotopic in situ hybridisation, Genet. Anal. Techn. Appl. 8, 24-35.

Moers, M. H. P., Gaub, H. E., and van Hulst, N. F. (1994) Poly(diacetylene) monolayers studied with a fluorescence scanning near-field optical microscope, Langmuir 10, 2774-2777.

Moers, M. H. P., Kalle, W. H. J ., Ruiter, A. G. T., Wiegant, J. C. A. G., Raap, A. K., Greve, J ., de Grooth, B. G., and van Hulst, N. F. (1996) Fluorescence in situ hybridisation on metaphase chromosomes observed by near-field microscopy, J . Microsc. 182, 40- 45.

van Noort, S. J. T., van der Werf, K. O., de Grooth, B. G., van Hulst, N. F., and Greve, J . (1997) Height anomalies in tapping mode atomic force microscopy in air, UItramicroscopy. [I n press]

Pietrasanta, L. I., Schaper, A., and J ovin, T. M. (1994) Probing specific molecular conformations with the scanning force microscope: Complexes of plasmid DNA and anti-Z-DNA antibodies, Nucleic Acids Res. 22, 3288-3292.

Pohl, D. W., Denk, W., and Lanz, M. (1984) Optical stethoscopy: Image recording with resolution $\lambda / 20$, Appl Phys Lett. 44, 651-653.

Putman, C. A. J ., de Grooth, B. G., Wiegant, J ., Raap, A. K., van der Werf, K. O., van Hulst, N. F., and Greve, J . (1993) Detection of in situ hybridisation to human chromosomes with the atomic force microscope, Cytometry 14, 356-361.

Putman, C. A. J ., van der Werf, K. O., de Grooth, B. G., van Hulst, N. F., and Greve, J . (1994) Viscoelasticity of living cells allows high resolution imaging by tapping mode atomic force microscopy, Biophys. J . 67, 1749-1753.

Radmacher, M., Tillman, R. W., and Gaub, H. E. (1992) From molecules to cells-Imaging soft samples with AFM, Science 257, 1900-1905. [and references therein]

Radmacher, M., Fritz, M., Hansma, H. G., and Hansma, P. K. (1994) Direct observation of enzyme activity with the atomic force microscope, Science 265, 1577-1579.

Rudkin, G. T., and Stollar, B. D. (1977) High resolution detection of DNA-RNA hybrids in situ by indirect immunofluorescence, Nature 265, 472-473.

Ruiter, A. G. T., Veerman, J .-A., van der Werf, K. O., and van Hulst, N. F. (1997a) Dynamic behavior of tuning fork shearforce feedback, Appl. Phys. Lett. [In press]

Ruiter, A. G. T., Veerman, J -A., Garcia-Parajo, M. F., and van Hulst, N. F. (1997b) Single molecule rotational and translational diffusion observed by near-field scanning optical microscopy, J . Phys. Chem. [In press]

Schaper, A., Starink, J . P. P., and J ovin, T. M. (1994) The scanning force microscopy of DNA in air and in propanol using new spreading agents, FEBS Lett. 355, 91-95.

Schmidt, Th., Schutz, G. J ., Baumgartner, W., Gruber, H. J ., and Schindler, H. (1995) Characterization of photophysics and mobility of single molecules in a fluid lipid membrane, J. Phys. Chem. 99, 17662-17668.

Toledo-Crow, R., Yang, P. C., Chen, Y., and Vaez-I ravani, M. (1992) Near-field differential scanning optical microscope with atomic force regulation, Appl. Phys. Lett. 60, 2957-2959.

Tsien, R. Y., and Waggoner, A. (1990) in Pawley, J. B. (Ed.), Handbook of Biological Confocal Microscopy, Plenum, New York.

Vamosi, G., Gohlke, C., and Clegg, R. M. (1996) Fluorescence characteristics of 5-carboxy tetramethylrhodamine linked covalently to the $5^{\prime}$ end of oligonucleotides: Multiple conformers of single-stranded and double-stranded dye-DNA complexes, Biophys. J . 71, 972-994.

Wiegant, J., Kalle, W., Mullenders, L., Brookes, S., Hoovers, J . M. N., Dauwerse, J . G., van Ommen, G. J . B., and Raap, A. K. (1992) High-resolution in situ hybridisation using DNA halo preparations, Hum. Mol. Genet. 1, 587-591.

Wiegant, J ., Wiesmeijer, C. C., Hoovers, J . M. N., Schuuring, E., d'Azzo, A., Vrolijk, J., Tanke, H. J., and Raap, A. K. (1993) Multiple and sensitive fluorescence in situ hybridisation with rhodamine, fluorescein- and coumarin-labelled DNAs, Cytogenet. Cell Genet. 63, 73-76.

Wyman, C., Grotkopp, E., Bustamante, C., and Nelson, H. C. M. (1995) Determination of heat-shock transcription factor 2 stoichiometry at looped DNA complexes using scanning force microscopy, EMBO J . 14, 117-123.

Wyman, C. (1997) Private communication.

Xie, X. S., and Dunn, R. C. (1994) Probing single molecule dynamics, Science 265, 361-364. 